\title{
ENFERMEDAD RENAL DIABÉTICA EN PACIENTES PEDIÁTRICOS
}

\section{DIABETIC KIDNEY DISEASE IN PEDIATRIC PATIENTS}

\author{
Valeria Hirschler
}

\section{RESUMEN}

La albuminuria es un marcador temprano de enfermedad renal; su diagnóstico se define cuando el rango de albumina/creatinina es mayor de $30 \mathrm{mg} / \mathrm{g}$ y persiste en al menos dos de tres determinaciones, igual que en la población adulta. Las conclusiones de varios estudios pediátricos son contradictorias; algunos hallan asociación entre obesidad, factores de riesgo cardiovascular y albuminuria y otros no. Se sugiere que la excreción urinaria de albúmina podría estar aumentada en los niños normopeso vs los obesos lo cual podría deberse al incremento de la actividad física en los niños normopeso respecto de los niños obesos. Un estudio en adolescentes con diabetes tipo 2 observó que aquellos que presentaban albuminuria desarrollaban insuficiencia renal en la adultez temprana. En la diabetes tipo 1 se recomienda el screening para microalbuminuria anualmente después de los 10 años de edad o a los 5 años del comienzo de la enfermedad y en diabetes tipo 2 al momento del diagnóstico.

Palabras clave: población pediátrica, enfermedad renal, albuminuria, obesidad, diabetes tipo 1, diabetes tipo 2.

Revista de la Sociedad Argentina de Diabetes 2017; Vol. 51 (120-122)

\begin{abstract}
Albuminuria is an early marker of the kidney disease, its diagnosis is defined when the range of albumin/creatinine is greater than $30 \mathrm{mg} / \mathrm{g}$ and persists in at least two or three determinations like in the adult population. The conclusions of various pediatric studies are contradictory, some find associations between obesity, cardiovascular risk factors and albuminuria and others not. It is suggested that urinary albumin excretion may be increased in children with normal weight vs obese children, likely caused by increased physical activity in children with normal weight compared with obese children. A study in adolescents with type 2 diabetes, observed that those presenting albuminuria, developed kidney insufficiency in the early adulthood. In type 1 diabetes, screening is recommended for microalbuminuria annually after 10 years old or at 5 years from the onset of disease and in type 2 diabetes at the moment of diagnosis.
\end{abstract}

Key words: pediatric population, kidney disease, albuminuria, obesity, type 1 diabetes, type 2 diabetes.

Revista de la Sociedad Argentina de Diabetes 2017; Vol. 51 (120-122)
Hospital Durand, Servicio de Nutrición; miembro del Comité de Nefropatía de la Sociedad Argentina de Diabetes, CABA, Argentina

Contacto de la autora: Valeria Hirschler

E-mail: vhirschler@gmail.com
Correspondencia: Maipú 812 (C1006ACL) CABA, Argentina Tel: (011) 4801-8387

Fecha de trabajo recibido: $18 / 08 / 17$

Fecha de trabajo aceptado: 30/08/17

Conflictos de interés: la autora declara que no existe conflicto de interés

\section{Enfermedad renal diabética en la población infantil}

El desarrollo de enfermedad crónica renal es generalmente la culminación de la interacción de diversos factores de riesgo. La albuminuria es un marcador temprano de enfermedad renal; su diagnóstico se define cuando el rango de albumina/creatinina es mayor de 30 $\mathrm{mg} / \mathrm{g}$ y persiste en al menos dos de tres determinaciones, igual que en la población adulta.

La obesidad representa un ejemplo de estado de multitoxicidad asociado a una mayor susceptibilidad genética y/o reducción del número de nefrones. Por estas razones la obesi- dad puede iniciar una remodelación renal y/o acelerar la falla renal'. En los adultos la obesidad es un factor de riesgo de albuminuria y por lo tanto de enfermedad renal. Sin embargo, pocos estudios han examinado la asociación de obesidad y diabetes tipo 2 en el desarrollo de proteinuria en la población general infantil.

Estudios pediátricos examinaron la asociación entre factores de riesgo cardiovascular y albuminuria llegando a conclusiones contradictorias. Distintos estudios encontraron una relación positiva entre obesidad y albuminuria en la población pediátrica ${ }^{2,3}$. Uno de ellos comparó 79 niños normo-peso con 86 niños 
obesos de 13 años de edad y observó que la microalbuminuria se asociaba a obesidad, indicando una probable disfunción glomerular y tubular temprana como consecuencia de la obesidad $^{2}$. Coincidente con este resultado, un trabajo realizado en 588 niños obesos demostró que los niños con síndrome metabólico presentaban mayor prevalencia de microalbuminuria que aquellos sin síndrome metabóli$\mathrm{CO}^{3}$. Sin embargo, estos estudios se realizaron en población hospitalaria y fueron estudios transversales. Por el contrario, el estudio $\mathrm{Na}$ tional Health and Nutrition Examination Survey (NHANES) 1999-20044, realizado en Estados Unidos en la población general, demostró que los adolescentes obesos tenían menor prevalencia de microalbuminuria que los no obesos. De acuerdo con estos resultados, un estudio efectuado en nuestro país evaluó la albuminuria en 1.564 alumnos de 9 años de edad en escuelas primarias de la Ciudad de Buenos Aires, y demostró que la presencia de sobrepeso y obesidad fue un factor protector de los niveles elevados de albuminuria $(\mathrm{OR}, 0,77[95 \% \mathrm{Cl}$ 0,64-0,92]) ajustado por variables confundentes ${ }^{5}$. Más aún, un estudio reciente en más de 11.000 chicos con diabetes tipo 1 y sobrepeso demostró que los chicos con obesidad presentaban niveles menores de microalbuminuria que aquellos con normopeso (OR 0,6, 99\% $\mathrm{Cl} 0,4-1,0)^{6}$. La explicación de estos hallazgos no se conoce. Se sugiere que podría deberse al incremento de actividad física en los niños normopeso vs los que presentan sobrepeso u obesidad. Los niños normopeso son generalmente más activos que los obesos y como la albuminuria se asocia a la actividad física, la excreción urinaria de albúmina podría estar aumentada en los niños normopeso vs los obesos. Debido a la dificultad de cuantificar la actividad física en la población pediátrica, ya que gran parte de ésta es no programada, es que probablemente se observó una asociación inversa entre microalbuminuria y obesidad en niños. Estos resultados sugieren que los niveles de albuminuria en niños aparentemente sanos no sería una herramienta útil para la identifica- ción temprana de niños con riesgo de diabetes tipo 2. Deberían realizarse investigaciones longitudinales y randomizadas para confirmar estos hallazgos.

Con respecto a la asociación de albuminuria y diabetes tipo 2 los estudios también son muy controvertidos y difieren sus resultados. Es importante recordar que la diabetes tipo 2 fue, hasta hace pocas décadas, una enfermedad propia de la edad adulta. Sin embargo, con el incremento de la obesidad, la diabetes tipo 2 pasó a formar parte de las enfermedades pediátricas. Por estas razones, el número de pacientes pediátricos con diabetes tipo 2 es aún pequeño. En una revisión realizada en Canadá en 90 chicos con diabetes tipo 2 se detectó que 14 de 90 chicos (16\%) presentaban macroalbuminuria a los ocho años del diagnóstico de diabetes tipo $2^{7}$. Se realizaron 10 biopsias y se observó que en 9 de 10 biopsias fueron por enfermedad renal compleja autoinmune o glomeruloesclerosis y ninguno por nefropatía clásica de la diabetes ${ }^{7}$. Los autores concluyeron que el diagnóstico de nefropatía diabética en chicos con diabetes tipo 2 no puede determinarse por clínica o laboratorio solamente, sino también debe confirmarse con biopsia? Por otro lado, se realizó un estudio en adolescentes de 15 años con diabetes tipo 2 y se observó que 34\% presentaba microalbuminuria ${ }^{8}$. Además los adolescentes con rango albumina/creatinina en el mayor tertil presentaban menor insulino-sensibilidad ${ }^{8}$. Incluso un estudio realizado en Canadá en una población de más de 300 adolescentes con diabetes tipo 2 se observó que el 9,1\% de los pacientes que presentaba albuminuria en la adolescencia desarrollaba insuficiencia renal en la adultez temprana9. Sin embargo, pese al incremento de la prevalencia de diabetes tipo 2 en pediatría, aún no hay estudios longitudinales y randomizados de esta patología en este grupo etareo para confirmar estos hallazgos.

En la diabetes tipo 1, la Asociación Americana de Diabetes recomienda el screening para microalbuminuria anualmente después de los 10 años de edad o a los 5 años del comienzo de 
la enfermedad. El diagnóstico requiere dos de tres test positivos para albuminuria en muestras de orina al azar ${ }^{10}$. El diagnóstico temprano de microalbuminuria en niños diabéticos tipo 1 es importante ya que existe un tratamiento efectivo para limitar la progresión de nefropatía diabética 9 . Un estudio que comparó 368 adolescentes que no presentaban diabetes con 175 adolescentes con diabetes, se observó que la excreción de albumina fue significativamente menor en los adolescentes sin diabetes $(4,0 \mu \mathrm{g} / \mathrm{min})$ que en aquellos con diabetes tipo $1(5,0 \mu \mathrm{g} / \mathrm{min})^{10}$. En un estudio realizado en 7.549 diabéticos tipo 1, menores de 20 años de edad y con más de un año de evolución de diabetes, se detectó microalbuminuria persistente en 329 participantes $(4,4 \%)^{12}$. La mayor frecuencia se asoció al tiempo de duración, al incremento de la HbA1c, a la mayor edad, al sexo femenino, a la mayor tensión arterial y al menor IMC. La prevalencia de microalbuminuria fue poco frecuente $(<2 \%)$ en aquellos participantes con $\mathrm{HbA} 1 \mathrm{c}<7,5 \%$. Los autores concluyeron que hubo una menor prevalencia de nefropatía en aquellos participantes con mejor control de glucemia y de tensión arterial ${ }^{11}$. Esto enfatiza la importancia de un screening en los pacientes pediátricos con diabetes tipo 1 para asegurar el diagnóstico precoz de microalbuminuria y evitar las complicaciones tempranas ${ }^{11}$. Los exámenes de orina deben realizarse con una muestra de orina al azar cada seis meses e incentivar al paciente para que optimice los controles de glucemia y normalice la tensión arterial. El tratamiento indicado en pediatría se realiza con IECA.

Como conclusión, debería focalizarse el screening en pacientes diabéticos tipo 1 de más de 5 años de evolución para realizar un diagnóstico temprano y evitar la progresión de la enfermedad renal. En el caso de diabetes tipo 2 los resultados son controvertidos, sin embargo el screening de albuminuria debería realizarse al diagnóstico de la misma. Deberían efectuarse estudios longitudinales y randomizados en este grupo etario para confirmar estos hallazgos.

\section{BIBLIOGRAFÍA}

1. Cignarelli M, Lamacchia O. Obesity and kidney disease. Nutr Metab Cardiovasc Dis. 2007; 17(10):757-62.

2. Csernus K, Lanyi E, Erhardt E, et al. Effect of childhood obesity and obesity-related cardiovascular risk factors on glomerular and tubular protein excretion. Eur J Pediatr 2005; 164(1):44-49.

3. Invitti $C$, Maffeis $C$, Gilardini L, et al. Metabolic syndrome in OB Caucasian children: prevalence using WHO-derived criteria and association with nontraditional cardiovascular risk factors. Int J Obes (Lond) 2006; 30:627-633.

4. Nguyen S, McCulloch C, Brakeman P, et al. Being overweight modifies the association between cardiovascular risk factors and microalbuminuria in adolescents. Pediatrics 2008; 121(1):37-45.

5. Hirschler V, Molinari C, Maccallini G, Aranda C. Is albuminuria associated with obesity in school children? Pediatr Diabetes 2010; 11(5):322-30.

6. Redondo MJ, Foster NC, Libman IM, et al. Prevalence of cardiovascular risk factors in youth with type 1 diabetes and elevated body mass index. Acta Diabetol 2016; 53(2):271-7.

7. Sellers EA, Blydt-Hansen TD, Dean HJ, Gibson IW, Birk $\mathrm{PE}$, Ogborn M. Macroalbuminuria and renal pathology in First Nation youth with type 2 diabetes. Diabetes Care 2009; 32(5):786-90.

8. Bjornstad P, Maahs DM, Cherney DZ, et al. Insulin sensitivity is an important determinant of renal health in adolescents with type 2 diabetes. Diabetes Care 2014; 37(11):3033-9.

9. Dart $A B$, Sellers EA, Martens PJ, et al. High burden of kidney disease in youth-onset type 2 diabetes. Diabetes Care 2012; 35:1265-71.

10. American Diabetes Association. Standards of medical care in diabetes 2012. Diabetes Care 2012; 35 (Suppl. 1):S11-S63.

11. Rademacher E, Mauer M, Jacobs DR Jr, et al. Albumin excretion rate in normal adolescents: relation to insulin resistance and cardiovascular risk factors and comparisons to type 1 diabetes mellitus patients. Clin J Am Soc Nephrol 2008; 3:998-1005.

12. Daniels M, DuBose SN, Maahs DM, et al. Factors associated with microalbuminuria in 7,549 children and adolescents with type 1 diabetes in the T1D Exchange clinic registry. Diabetes Care 2013; 36:2639-45. 